negative disposition, weaker support system, poor problemsolving skills, unclear on their healthcare role, significant knowledge gaps, and poor self-management skills. We report here on the baseline data. Descriptive statistics were performed using Wilcoxon Mann Whitney Test, Exact Pearson Chi Square Test, or a Pearson Chi Square Test, as appropriate. Results We sent letters to 1916 patients affiliated with 10 CaNIOS centers in January 2018. A total of 571 patients responded and $541(28.2 \%)$ completed all the baseline questionnaires. The average age was 50 (range 19-85), 93\% were female with an average duration of lupus of 17 years. Three quarters of respondents were Caucasian, 55\% had a college or university degree and 64\% were working while 26\% reported being on disability. We report in table 1 the self-reported disease characteristic at baseline using the Systemic Lupus Activity Questionnaire (SLAQ), the Lupus Damage Index Questionnaire (LDIQ) and the physical and mental component summary scores of the SF-36 $6^{\mathrm{TM}}$, the number of computer hour usage, the frequency of searching for medical information and the PAM. We can observe that one third (35\%) of our patients have a low PAM score at baseline.

Conclusions We observed low patient self-management ability in one third of a large sample of lupus patients participating into a trial of MyLupusGuide suggesting that this vulnerable population needs additional resources to improve their confidence in self-managing their lupus.

\begin{tabular}{|c|c|c|c|c|c|}
\hline Characteristic & $\begin{array}{l}\text { Group A } \\
(n=265)\end{array}$ & $95 \% \mathrm{Cl}$ & $\begin{array}{l}\text { Group B } \\
(n=276)\end{array}$ & $95 \% \mathrm{Cl}$ & $p$-value \\
\hline \multicolumn{6}{|l|}{ SLAQ } \\
\hline Mean (sd) & $13.0(7.6)$ & $\begin{array}{l}(12.1 ; \\
14.0)\end{array}$ & $14.9(8.3)$ & $\begin{array}{l}(14.0 ; \\
15.9)\end{array}$ & $0,0125^{*}$ \\
\hline \multicolumn{6}{|l|}{ LDIQ } \\
\hline $\begin{array}{l}\text { Mean (sd) } \\
\text { SF36 }\end{array}$ & $3.3(2.9)$ & $(3.0 ; 3.7)$ & $3.7(3.2)$ & $(3.4 ; 4.1)$ & 0,0952 \\
\hline PC mean (sd) & $40.2(11.5)$ & $\begin{array}{l}(38.8 ; \\
41.6)\end{array}$ & $37.7(12.2)$ & $\begin{array}{l}(36.3 ; \\
39.2)\end{array}$ & $0,0266^{*}$ \\
\hline MC mean (sd) & $44.4(11.2)$ & $\begin{array}{l}(43.0 ; \\
45.7)\end{array}$ & 43.6 (11.4) & $\begin{array}{l}(42.3 ; \\
45.0)\end{array}$ & 0,4103 \\
\hline \multicolumn{6}{|c|}{ Computer usage (hours/week) } \\
\hline Mean (sd) & $15.5(15.8)$ & $\begin{array}{l}(13.6 ; \\
17.5)\end{array}$ & $13.4(11.3)$ & $\begin{array}{l}(12.1 ; \\
14.8)\end{array}$ & 0,1184 \\
\hline \multicolumn{6}{|c|}{$\begin{array}{l}\text { Frequency of searching for medical } \\
\text { information (\%) }\end{array}$} \\
\hline Never & $6(2)$ & & $12(4)$ & & 0,2078 \\
\hline Sometimes & $154(59)$ & & $143(53)$ & & \\
\hline Frequently & $74(28)$ & & 91 (34) & & \\
\hline Very frequently & $28(11)$ & & $23(9)$ & & \\
\hline PAM & & & & & 0,7788 \\
\hline Mean (sd) & $61.1(13.6)$ & $\begin{array}{l}(59.4 ; \\
62.7)\end{array}$ & $61.1(14.0)$ & $\begin{array}{l}(59.4 ; \\
62.7)\end{array}$ & \\
\hline Level 1 n (\%) & $39(14.7)$ & & $49(17.8)$ & & \\
\hline Level 2 n (\%) & $57(21.5)$ & & $50(18.2)$ & & \\
\hline Level 3 n (\%) & $111(41.9)$ & & $114(41.5)$ & & \\
\hline Level 4 n (\%) & $58(21.9)$ & & $62(22.5)$ & & \\
\hline
\end{tabular}

Acknowledgements We thank the Canadian Institutes for Health Research and their Knowledge-to-Action grant program for supporting this trial. We also thank the individual CaNIOS centers and their local investigators, research assistants and patients that have made this study possible.

Trial registration The trial registration numbers are NCT02950714 and KAL-356634.

\section{LL-08 WALK SLE - END OF STUDY RESULTS OF A PILOT STUDY EXPLORING WALK WITH EASE, A SELF-DIRECTED WALKING PROGRAM, IN LUPUS PATIENTS}

${ }^{1}$ Brittaney-Belle E Gordon, ${ }^{2}$ Katherine Kaufman, ${ }^{1}$ Sean T Hicks, ${ }^{1}$ Rebecca J Cleveland, ${ }^{1} J u l i e$ A Norfleet, 'Leigh F Callahan, 'Saira Z Sheikh*. 'University of North Carolina at Chapel Hill School of Medicine, Chapel Hill, NC, USA; ${ }^{2}$ Duke University School of Medicine, Durham, NC, USA

\subsection{6/lupus-2018-Ism.118}

Background Fatigue and arthritis are common sources of impairment in SLE patients that persist despite lifestyle and pharmacologic interventions. Walk with Ease (WWE) is the Arthritis Foundation's 6 week evidence-based walking program developed for adults with arthritis that has been shown to improve physical function, pain, stiffness and fatigue in individuals with arthritis. WWE is offered in two formats - group (instructor led) or self-directed. The goal of this study was to examine the feasibility of self-directed WWE in SLE patients.

Methods We enrolled 75 SLE patients to take part in the WWE program, all of whom completed an initial evaluation. The post- 6 week evaluation with no usual care comparison group was conducted in 46 patients. Self-reported outcomes including symptoms (pain, stiffness and fatigue visual analog scales [VAS]) and Functional Assessment of Chronic Illness Therapy-Fatigue (FACIT-fatigue) scale were evaluated. Means and effect sizes (ES) with 95\% confidence intervals were computed for changes in symptoms between baseline and 6 weeks, adjusted for gender, age, race, and baseline outcome. Participant satisfaction measures with WWE are reported as percentages.

Results Participants were mainly women (96.0\%), aged $\geq 40$ years, self-identified as black (53.3\%) and 13 years from initial SLE diagnosis. Fifteen (20.0\%) participants also had a physician diagnosis of OA, while $21(28.0 \%)$ participants had concurrent fibromyalgia. Four participants were screen failures, 9 were withdrawn by the study team, 13 were lost to follow-up and 3 had not yet reached the end of the 6 week protocol. There were 46 participants who completed the WWE program; no significant differences in baseline measures were observed between those who did and did not complete the study. At the end of 6 weeks, participants reported having reduced FACIT-fatigue scores $(\mathrm{ES}=0.14)$, as well as having reduced scores for VAS fatigue and stiffness $(\mathrm{ES}=0.22$ and 0.11 , respectively [table 1]). Most participants reported walking for at least 30 minutes/day (47.7\%), $\geq 3$ days/week $(86.4 \%)$ and utilized the workbook for motivation (65.3\%). Participants also reported increased physical activity (81.8\%), confidence to continue exercising (97.8\%) and satisfaction from the program $(97.8 \%)$. 
Abstract LL-08 Table 1 Effect Sizes and means (SD) for overall effectiveness of WWE from baseline and follow-up

\begin{tabular}{llll}
\hline Outcome measures & Baseline mean(SD) & Follow-up mean(SD) & Effect size \\
\hline FACIT Fatigue & $24.1(29.4)$ & $19.8(30.4)$ & 0.14 \\
VAS Fatigue & $49.26(71.19)$ & $33.53(71.16)$ & 0.22 \\
VAS Pain & $31.58(54.11)$ & $28.98(53.27)$ & 0.05 \\
VAS Stiffness & $40.25(65.79)$ & $33.33(63.74)$ & 0.11 \\
\hline
\end{tabular}

Conclusions WWE is a feasible, low-cost program that may decrease SLE-related fatigue and stiffness, improve activity and promote long-term lifestyle changes.

Trial registration ClinicalTrials. gov Identifier: NCT02631005.

\section{LL-09 CHILDHOOD ARTHRITIS AND RHEUMATOLOGY RESEARCH ALLIANCE/LUPUS FOUNDATION OF AMERICA CHILDHOOD ONSET SYSTEMIC LUPUS ERYTHEMATOSUS RESEARCH PRIORITIZATION SURVEY}

1,25tacy P Ardoin* ${ }^{3}$ Lyna Merzog, 1,3Aimee Hersh. ${ }^{1}$ Childhood Arthritis and Rheumatology Research Association, USA; ${ }^{2}$ Departments of Pediatrics and Internal Medicine, Ohio State University, USA; ${ }^{3}$ Lupus Foundation of America, USA; ${ }^{4}$ Department of Pediatrics, University of Utah, USA

\subsection{6/lupus-2018-Ism.119}

Background Compared to adult-onset SLE, childhood onset SLE (cSLE) tends to present with more severe disease and more widespread organ involvement. Additionally, cSLE associated with higher mortality and damage accrual. However, despite the severity of the disease few commonly used SLE medications have been rigorously studied in children, and important knowledge gaps remain concerning the epidemiology, natural history, pathophysiology, genetics and optimal treatment of cSLE. In order to identify high priority areas for future research in cSLE, the Lupus Foundation of America (LFA) and the Childhood Arthritis and Rheumatology Research Alliance (CARRA) partnered to perform and cSLE research prioritization survey.

Methods Using information from the 2013 CARRA Lupus Group Research Prioritization Exercise and the most recent LFA Parent and Caregiver Survey, we identified areas of clinical and research need in cSLE. A work group was assembled to develop and refine survey questions which ask clinicians and researchers to rank research topics that are most urgent advance patient outcomes. The survey was programmed using Qualtrics software. The survey was beta tested by a separate group of clinicians and researchers. The proposed survey administration was deemed not to be human subjects research by the University of Utah and Nationwide Children's Institutional Review Boards. Descriptive statistics will be used to analyze survey responses.

Results The survey will be administered to members of CARRA and also to collaborators in the inflammatory skin disease interest group of the Pediatric Dermatology Research Association (PeDRA) and the glomerulonephritis interest group of the Midwestern Pediatric Nephrology Consortium (MWPNC). Together, these organizations include over 800 clinicians and researchers.

Conclusions The results of the CARRA/LFA Research Prioritization Survey will be used to guide funding programs for
cSLE within the Lupus Foundation of America, to share with potential donors, and to use in advocacy efforts promoting state and federal funding for cSLE.

Acknowledgements This study is supported by funding the Lupus Foundation of America and the Childhood Arthritis and Rheumatology Research Alliance.

\section{Tissue Damage}

\section{TD-01 NOVEL IL-16/MIR-125 AXIS CONTROLS NEUTROPHIL RECRUITMENT IN PRISTANE-INDUCED LUNG INFLAMMATION}

${ }^{1} S$ Smith, ${ }^{2} \mathrm{DJ}$ Wallace ${ }^{*},{ }^{1,2} \mathrm{CA}$ Jeffries. ${ }^{1}$ Royal College of Surgeons, Dublin, Ireland; ${ }^{2}$ CedarsSinai Medical Center, Los Angeles, CA

\subsection{6/lupus-2018-Ism. 120}

Background Severe lung inflammation and alveolar hemorrhage can be life threatening in lupus patients. Neutrophil influx is the key driver of this pathology. MiR-125a expression is decreased in patients with lupus pneumonitis. IL-16 is a novel target for mir-125a.

Methods Sera and monocytes from 42 SLE patients, 16 with pulmonary involvement was obtained. Gene expression profiles of monocytes from healthy controls and SLE patients were analyzed by qPCR and correlated with disease activity and organ involvement. Monocytes were transfected with mir-125a mimics and antagomirs and effects on IL-16 mRNA determined by qPCR. Biotin-labelled mir-125a mimics were immunoprecipitated from monocytes and IL-16 confirmed as a direct target by qPCR. Autoimmune lung inflammation was measured following intraperitoneal injection of pristane followed by FACs and qPCR analysis of cell populations in the lung.

Results Reduced miR-125a and enhanced IL-16 expression in SLE patient monocytes was observed and found to correlate with lung involvement. Furthermore, in the pristane model of acute 'SLE-like' lung inflammation, and alveolar hemorrhage, we observe reduced pulmonary miR-125a and enhanced IL-16 expression. Neutrophil infiltration was markedly reduced in the peritoneal lavage of pristine-treated IL-16 deficient mice and elevated following intranasal delivery of IL-16. Moreover, a miR-125a mimic reduces pristane-induced IL-16 expression and neutrophil recruitment, and rescues lung pathology. Mechanistically, IL-16 acts directly on the pulmonary epithelium and markedly enhances neutrophil chemoattractant expression both in vitro and in vivo, whilst the miR-125a mimic can prevent this.

Conclusions A miR-125a mimic reduces pristane-induced IL-16 expression and neutrophil recruitment, and rescues lung pathology. Mechanistically, IL-16 acts directly on the pulmonary epithelium and markedly enhances neutrophil chemoattractant expression both in vitro and in vivo, while the miR-125a mimic can prevent this. A novel role for miR-125a/IL-16 in regulating lung inflammation suggests that this axis may be a therapeutic target for management of acute lung injury in SLE.

Acknowledgement Support provided by NIH Grant (HL0127384), Lupus Research Allliance (ALR/TIL332436), Irish Research Council (GO1PD/2014/512). 